\title{
LES POLONAIS EN RUSSIE ET AU KAZAKHSTAN
}

À l'inverse de ce qui s'est passé dans les autres pays situés à l'Est de la Pologne ${ }^{1}$, la grande majorité des Polonais habitant la Russie et le Kazakhstan s'y est trouvée contre son gré. La Sibérie et le Kazakhstan ont été peuplés de force, dès leur annexion par le pouvoir tsariste. Les premiers Polonais arrivent en Sibérie au début du XVII ${ }^{\mathrm{e}}$ siècle; il s'agit de 1550 prisonniers de guerre. Dès le premier partage de la Pologne, en 1772, les déportations commencent; jusqu'à la fin du XVII ${ }^{\mathrm{e}}$ siècle, elles englobent entre 5 et 10000 personnes. Au XIX ${ }^{\mathrm{e}}$ siècle, les répressions s'intensifient, surtout après les insurrections de 1830 et 1863, 250000 Polonais sont déportés en Sibérie et en Extrême-Orient. D'autres émigrent pour des raisons économiques et s'établissent dans les villes de Russie, surtout à SaintPétersbourg, oà en 1869 ils sont 11157 (1,6\% de la population).

D'après le premier recensement de 1897, 1174744 personnes déclaraient la nationalité polonaise. Ils étaient les plus nombreux dans les terrains constituant jadis les confins orientaux de la Pologne (885 952 personnes); dans les territoires de la Russie actuelle ils étaient au nombre de 186 259, dont 29177 en Sibérie; 25117 Polonais habitaient le Caucase, 11576 - l'Asie Centrale.

En 1914, le nombre de Polonais en Russie actuelle s'élevait à 288800 , en 1926, d'après le recensement soviétique - 204000 , dont 46000 en Sibérie, oà ils constituaient $5,2 \%$ de la population $(9,1 \%$ dans le gouvernorat de Ienissey, $14,2 \%$ dans celui d'Irkoutsk). Dans les années trente de nombreux Polonais habitant la Biélorussie et l'Ukraine sont déportés en Russie et au Kazakhstan; en 1936, sur 200000 personnes déportées de la région de Podole, la moitié seulement survit au premier hiver kazakh.

Après l'invasion soviétique de 1939, 250000 soldats polonais sont envoyés au Nord et à l'Est de la Russie. Des déportations massives sont organisées dans les territoires annexés. Les pertes s'élèvent à environ 415000 victimes. En 1942, on dénombre 1692000 déportés, dont 203000

1 cf. "Les Polonais en Lituanie et en Lettonie" et "Les Polonais en Biélorussie et en Ukraine", dans les n ${ }^{\text {os }}$ 6/1994 et 7/1996 de Miscellanea Geographica. 
en Sibérie, 50000 dans l'Oural, 144500 au Kazkhstan, 102000 en Asie Centrale, 25000 dans le Caucase. Parmi eux, 462913 personnes parviennent à quitter l'Union Soviétique en 1942, avec l'armée Anders.

De nouvelles répressions et déportations ont lieu en 1944-45, lorsque les Soviétiques entrent dans les territoires polonais et $\mathrm{y}$ installent un gouvernement communiste. Peu de déportés regagnent la Pologne; ils constituent environ $10 \%$ de ceux qui, avant la guerre, étaient citoyens polonais.

D'après les recensements, le nombre de personnes déclarant la nationalité polonaise, a été le suivant:

\begin{tabular}{l|r|r|r|r}
\hline & \multicolumn{1}{|c|}{1959} & \multicolumn{1}{|c|}{1970} & \multicolumn{1}{|c|}{1979} & \multicolumn{1}{c}{1989} \\
\hline Russie & 118422 & 107084 & 99733 & 94596 \\
St-Pétersbourg et environs & 11662 & 10948 & 9607 & $7955^{1}$ \\
Moscou et environs & 10195 & 10496 & 7928 & $6920^{1}$ \\
Circonscription de Kaliningrad & & 4028 & 4245 & 4287 \\
Circonscription d'Irkoutsk & & 5200 & 4540 & 3118 \\
Karélie & & 2274 & 4077 & \\
Kazakhstan & 53102 & 61445 & 61136 & 59956 \\
Circonscription de Koktchetav & 25232 & 27062 & 25586 & 25400 \\
Circonscription d'Aqmola & 10459 & 10764 & 10600 & \\
\hline
\end{tabular}

${ }^{1}$ ville seulement

Ces chiffres sont inférieurs à la réalité, car les Polonais étaient nombreux à cacher leur nationalité, par peur de répressions, ou parce qu'ils espéraient ainsi avoir de meilleurs conditions d'existence. De plus, dans certaines circonscriptions, surtout là oà ils vivaient dispersés, la nationalité polonaise n'était pas évoquée. Le recensement de 1989 est, de ce point de vue, le plus complet. Ainsi, p.ex., on a noté 3485 Polonais dans la circonscription de Tioumen, 2626 - dans celle de Sverdlovsk (Iekaterinbourg), 2315 - de Rostov, 2081 - dans le Komi, 1926 - dans la ville de Kazan, 1704 - à Omsk.

Bien sûr, le nombre des Polonais diminue constamment: la russification est très forte, les mariages mixtes sont nombreux, les écoles manquent. En $1959,18,7 \%$ de Polonais déclaraient la langue polonaise comme leur langue maternelle contre seulement $15,1 \%$ en 1989. La grande majorité habite les villes $(73,2 \%$ en $1959,80,7 \%$ en 1970) - surtout St- Pétersbourg et Moscou, oà ceux qui y habitaient déjà avant 1917 , ou leur descendants sont encore nombreux. Il en est ainsi à Rostov. Dans la région de Kaliningrad les Polonais sont arrivés dans les années soixante-dix, de Lituanie et de Biélorussie. Partout ailleurs il s'agit des déportés ou leur descendants.

$\mathrm{Au}$ Kazakhstan les Polonais n'étaient que 4050 en 1926. Les déportés des années trente ont été disposés le long de la voie ferrée Krasnoarmeïsk - Alma Ata, dans les endroits inhabités, avec interdiction de les quitter. Actuellement ils habitent donc les terrains ruraux (72,1\% en $1959,59,2 \%$ en 1979), au Nord de la république. D'après les organisations locales, leur 
nombre dépasse les 100000 (38 000 dans la circonscription de Koktchetav, 33000 dans celui d'Aqmola), il est donc beucoup plus élevé que celui des données officielles. En effet, là aussi il n'était pas prudent de reconnaître la nationalité polonaise, et la russification était importante. En 1959, 24,1\% des Polonais déclaraient la langue polonaise comme étant leur langue maternelle, en 1989 - 12,2\% seulement. Actuellement, leur situation est d'autant plus grave, qu'ils ne connaissent pas la langue kazakh, devenue la langue officielle.

Après la chute du communisme et la dissolution de l'Union Soviétique, les Polonais sont devenus beaucoup plus actifs. Ils reconnaissent leur nationalité. Plusieurs organisations ont été créées dans de nombreuses villes de Russie, ainsi qu'à Koktchetav, Aqmola, Karaganda et Almaty au Kazakhstan. Des écoles polonaises ont été fondées à St-Pétersbourg, à Moscou, à Irkoutsk, dans 39 localités au Kazakhstan. La Gazeta Polska est éditée à Moscou.

Le rôle de l'église catholique est très important dans cette rennaissance, bien que certains conflits soient apparus, les autorités russes et kazakhs n'étant pas favorables à l'utilisation de la langue polonaise.

Ce regain se heurte à des difficultés dues surtout à la dispersion des Polonais dans ces énormes pays et à la faiblesse de leur contacts avec la Pologne dont l'aide se révèle insuffisante. Elle est pourtant nécessaire, surtout vis-à-vis des anciens déportés dont certain nombre voudraient revenir dans leurs régions d'origine situées d'ailleurs non seulement en Pologne, mais très souvent en Ukraine, en Biélorussie, en Lituanie ou en Lettonie. 
